\title{
Instability of the Perturbation Theoretical Chromodynamic Vacuum
}

\author{
Y.N. Srivastava, O. Panella \\ Physics Department 83 INFN, University of Perugia, Via Pascoli, Perugia Italy
}

A. Widom

Physics Department, Northeastern University, Boston MA, USA

\begin{abstract}
The standard model of strong interactions invokes the quantum chromodynamics (QCD) of quarks and gluons interacting within a fluid. At sufficiently small length scales, the effective interactions between the color charged particles within the fluid are thought to be weak. Short distance asymptotic freedom provides the perturbation theory basis for comparisons between QCD theory and laboratory high energy scattering experiments. It is here shown that the asymptotically free vacuum has negative dissipation implicit in the color electrical conductivity. Negative dissipation implies an asymptotically free QCD negative temperature excited state amplifier unstable to decay. The qualitative experimental implications of this instability are explored.
\end{abstract}

PACS numbers: 11.10.-z,11.15.-q,11.15.Tk,11.25.Db,11.55.Fv

The physical vacuum [1] in both quantum electrodynamics (QED) and quantum chromodynamics (QCD) is endowed with a radiation impedance, in Lorentzian units $R_{v a c} \equiv 1 / c$, an electric charge $e$ and a color charge $g$. The weak coupling of strength of QED and the strong coupling strength of QCD are described by the vacuum properties

$$
\begin{gathered}
\alpha=\left(\frac{R_{v a c} e^{2}}{4 \pi \hbar}\right)=\frac{e^{2}}{4 \pi \hbar c}, \\
\alpha_{s}=\left(\frac{R_{v a c} g^{2}}{4 \pi \hbar}\right)=\frac{g^{2}}{4 \pi \hbar c} .
\end{gathered}
$$

The quantum vacuum of both QED and QCD is far from being inert [2, 3, 4, 5] when probed at microscopic distances and times. For the relativistic wave vector $q=(\omega / c, \boldsymbol{k})$, one may speak of space-like vacuum correlation at an invariant wave number $Q \equiv \sqrt{-q^{2}}>0$ wherein

$$
Q^{2}=-q^{2}=|\boldsymbol{k}|^{2}-\left(\omega^{2} / c^{2}\right)
$$

One may also speak of time-like vacuum dynamics [6, 7] at an invariant frequency $\Omega \equiv c \sqrt{s}>0$ wherein

$$
s=q^{2}=\left(\omega^{2} / c^{2}\right)-|\boldsymbol{k}|^{2} .
$$


The complete vacuum dielectric $\varepsilon\left(Q^{2}\right)$, respectively color dielectric $\varepsilon_{s}\left(Q^{2}\right)$, response function and the complete magnetic permeability $\mu\left(Q^{2}\right)$, respectively color magnetic permeability $\mu_{s}\left(Q^{2}\right)$, describe a dynamic vacuum exhibiting charged, respectively color charged, fluctuating quantum currents. Both QED and QCD theory envision [8] a dynamic vacuum in terms of a $Q$-dependent coupling strength for space-like correlations

$$
\begin{gathered}
\alpha\left(Q^{2}\right)=\frac{e^{2}}{4 \pi \hbar c \varepsilon\left(Q^{2}\right)}=\frac{e^{2} \mu\left(Q^{2}\right)}{4 \pi \hbar c}, \\
\alpha_{s}\left(Q^{2}\right)=\frac{g^{2}}{4 \pi \hbar c \varepsilon_{s}\left(Q^{2}\right)}=\frac{g^{2} \mu_{s}\left(Q^{2}\right)}{4 \pi \hbar c} .
\end{gathered}
$$

Crucially important for what follows is that QED, respectively QCD, may also describe vacuum time-like dynamical fluctuations by employing an electrical conductivity $\sigma$, respectively a color electrical conductivity $\sigma_{s}$.

$$
\begin{aligned}
\varepsilon\left(s+i 0^{+}\right) & =1+\frac{i \sigma(s)}{c \sqrt{s}}, \\
\varepsilon_{s}\left(s+i 0^{+}\right) & =\frac{i \sigma_{s}(s)}{c \sqrt{s}} .
\end{aligned}
$$

Causality dictates dispersion relations between the dielectric response functions and the dynamic conductivities; Specifically

$$
\begin{gathered}
\varepsilon\left(Q^{2}\right)=1-\left(\frac{Q^{2}}{\pi c}\right) \int_{0}^{\infty} \frac{\Re e\{\sigma(s)\} d s}{s\left(s+Q^{2}\right) \sqrt{s}}, \\
\varepsilon_{s}\left(Q^{2}\right)=-\left(\frac{Q^{2}}{\pi c}\right) \int_{0}^{\infty} \frac{\Re e\left\{\sigma_{s}(s)\right\} d s}{s\left(s+Q^{2}\right) \sqrt{s}} .
\end{gathered}
$$

The central theorem of this work involves the nature of $\alpha\left(Q^{2}\right)$ and the $\alpha_{s}\left(Q^{2}\right)$ when analytically continued from the space-like $Q^{2}>0$ regime to the time-like $s>0$ regime wherein the complex coupling strengths are dynamic.

Theorem: For time-like wave vectors $s>0$, the sign of the real part of the conductivity is opposite to the sign of the imaginary part of the coupling strength.

Proof: Eqs.(4) and (5) imply

$$
\begin{aligned}
& -\Im m\left\{\alpha\left(s+i 0^{+}\right)\right\}=\left|\frac{\alpha\left(s+i 0^{+}\right)}{\varepsilon\left(s+i 0^{+}\right)}\right| \frac{\Re e\{\sigma(s)\}}{c \sqrt{s}}, \\
& -\Im m\left\{\alpha_{s}\left(s+i 0^{+}\right)\right\}=\left|\frac{\alpha_{s}\left(s+i 0^{+}\right)}{\varepsilon_{s}\left(s+i 0^{+}\right)}\right| \frac{\Re e\left\{\sigma_{s}(s)\right\}}{c \sqrt{s}} .
\end{aligned}
$$

from which the theorem follows.

(i) In standard QED perturbation theory, the vacuum conductivity obeys a condition of positive dissipation

$$
\Im m\left\{\alpha\left(s+i 0^{+}\right)\right\} \leq 0 \Rightarrow \Re e\{\sigma(s)\} \geq 0 .
$$


The vacuum heating induced by an applied electromagnetic field at high frequency is due to the creation of one or more pairs of oppositely charged particles and anti-particles.

(ii) In standard QCD perturbation theory, the vacuum color conductivity obeys a condition of negative dissipation, i.e. positive amplification

$$
\Im m\left\{\alpha_{s}\left(s+i 0^{+}\right)\right\} \geq 0 \Rightarrow \Re e\left\{\sigma_{s}(s)\right\} \leq 0 .
$$

Our purpose is to point out that negative dissipation in the form $\Re e\left\{\sigma_{s}(s)\right\}<0$ is implicit in all previously reported QCD perturbation analyses to finite order in $\alpha_{s}$.

The physical interpretation of QCD vacuum cooling induced by the application of a high frequency chromodynamic field is (at first glance) more than a little bit obscure. How might one cool what is already asserted to be the vacuum? A true quantum vacuum is conventionally visualized as having the lowest possible energy. No further cooling should then be possible. Asymptotic freedom [9, 10, 11] must refer to an excited state color amplifier. One may indeed spontaneously cool down an initially excited state. We have been forced to conclude that the asymptotically free QCD vacuum is in reality unstable to decay into the true vacuum state. However, the situation with regard to comparisons between theory and experiment may not be entirely desperate.

It is important to note that negative dissipation implicit in condensed matter electrical conductivity is by no means a new phenomenon in laboratory amplifying systems [12]. If some of the chemical substances within a condensed matter system have atoms or molecules in inverted populations of excited state electronic energy levels, then the electrical conductivity of the substance at hand (within an amplifier frequency bandwidth $\varpi$ ) can exhibit a negative dissipative part of the electrical conductivity, i.e. $\Re e\left\{\sigma_{\text {Amplifier }}(\omega \in \varpi)\right\} \leq 0$. Such systems [13, 14] exhibit a negative noise temperature $T_{n}(\omega \in \varpi)<0$ within the same bandwidth $\varpi$. The laboratory achievement of negative noise temperatures, characteristic of electronic energy levels with inverted populations, was absolutely essential in order to achieve the original maser and laser devices [15, 16, 17]. Negative noise temperatures also occur naturally in the astrophysical matter clouding galaxies [18, 19, 20, 21]. The negative dissipative part of the electrical conductivity in some astrophysical matter is made manifest via the measured negative absorption (i.e. amplification) of the electromagnetic radiation that passes through these materials. However, it must be realized that amplification due to excited state systems has only a finite lifetime. The excited state atoms decay into their ground states after giving rise to a burst of electromagnetic radiation. To again achieve the excited state amplifier status, energy must be pumped back into the matter by an external source.

We note that, Savvidy and Matinyan showed, a long time ago, that a constant color magnetic 
field is spontaneously generated in QCD [22, 23, 24, 25].

They interpreted it to imply an infrared instability inherent in QCD. On the other hand, the instability discussed in the present paper arises directly from the asymptotic freedom expression for the QCD coupling constant and is apparently quite different.

For the QCD problem at hand, let $\Lambda$ denote the wave number beyond which asymptotic freedom might be thought to prevail. Perturbation theory predictions [9, 10] for the QCD coupling strength appear in the form

$$
\frac{1}{\alpha_{s}\left(Q^{2}\right)}=\left[\frac{33-2 N_{f}}{12 \pi}\right] \ln \left[\frac{Q^{2}}{\Lambda^{2}}\right]
$$

wherein $N_{f}=5$ is the present experimental number of light quark flavors. The QCD question raised by us concerns the negative dissipative part of the color conductivity. We find from Eqs.(4), (77) and (10) that $\Re e\left\{\sigma_{s}(s)\right\}<0$ for $s>\Lambda^{2}$. The negative dissipation is implicit in all previous theoretical asymptotic freedom perturbation theory estimates. For both QED and QCD, the causal dispersion Eq.(6) yields conceptual problems for quantum field theory.

The vacuum screened Coulomb law potential energy between two charges, $Z_{1} e$ and $Z_{2} e$, may be written

$$
\begin{aligned}
U(r) & =\frac{Z_{1} Z_{2} e^{2}}{4 \pi r} \chi(r), \\
\chi(r) & =\frac{2}{\pi} \int_{0}^{\infty} \frac{\sin (Q r)}{Q \varepsilon\left(Q^{2}\right)} d Q .
\end{aligned}
$$

To achieve the Coulomb law of force between two point electrical charges one requires that

$$
\lim _{r \rightarrow \infty} \chi(r)=\lim _{Q^{2} \rightarrow 0} \frac{1}{\varepsilon\left(Q^{2}\right)}=1 .
$$

consistent with the dispersion Eq.(6) for $\varepsilon\left(Q^{2}\right)$. While the QED perturbation theory conductivity obeys the expected positive dissipation condition $\Re e\{\sigma(s)\} \geq 0$ in the time-like region $s>0$, the dispersion relation yields a change in sign for $\varepsilon\left(Q^{2}\right)$ in the space-like region $Q^{2}>0$. There exists a positive wave number $K>0$ for which $\varepsilon\left(K^{2}\right)=0$; i.e. there appears a Landau ghost [26, 27]. For space-like wave vectors $Q^{2}>K^{2}$, two particles whose electric charges have the same sign will tend to attract rather than repel one another. If within even a limited range of wave numbers attraction occurs between particles whose charges have the same sign, then there will be a strong tendency towards Cooper pairing [28] and superconductivity. The experimental QED vacuum is an insulator rather than a superconductor. One tends to regard the Landau ghost as an unphysical [29] theoretical QED embarrassment. 
The vacuum confining potential energy between two color matrix charged particles, $g \boldsymbol{\top}_{1}$ and $g \mathrm{\top}_{2}$, may be written[30]

$$
\begin{aligned}
& U_{s}(r)=\frac{\eta^{a b} T_{a 1} T_{b 2} g^{2}}{4 \pi r} \chi_{s}(r), \\
& \chi_{s}(r)=\frac{2}{\pi} \int_{0}^{\infty} \frac{\sin (Q r)}{Q \varepsilon_{s}\left(Q^{2}\right)} d Q .
\end{aligned}
$$

By taking two derivatives of Eq.(13),

$$
\chi_{s}^{\prime \prime}(r)=-\frac{2}{\pi} \int_{0}^{\infty} \frac{Q \sin (Q r)}{\varepsilon_{s}\left(Q^{2}\right)} d Q,
$$

one finds the finite limits

$$
\begin{aligned}
& \frac{L^{2}}{2}=\lim _{Q^{2} \rightarrow 0} \frac{\varepsilon_{s}\left(Q^{2}\right)}{Q^{2}}=-\left(\frac{1}{\pi c}\right) \int_{0}^{\infty} \frac{\Re e\left\{\sigma_{s}(s)\right\}}{s^{5 / 2}} d s, \\
& \frac{2}{L^{2}}=-\lim _{r \rightarrow \infty} \chi_{s}^{\prime \prime}(r)=-\lim _{r \rightarrow \infty} \frac{2 \chi_{s}(r)}{r^{2}},
\end{aligned}
$$

in agreement with the color dielectric function dispersion Eq. (6) Eqs.(13) and (15) is the linear confining potential

$$
U_{s}(r)=-\left(\eta^{a b} T_{a 1} T_{b 2}\right) \tau r \quad \text { as } \quad r \rightarrow \infty .
$$

Eq.(16) may be viewed as a potential due to a QCD color electric flux tube, i.e. a QCD string with tension

$$
\tau=\frac{g^{2}}{4 \pi L^{2}}
$$

connecting two color charged particles. The above argument is similar to that given by Feynman [31] who conjectured that the color confining potential should obey the bi-harmonic equation. The fact that the Landau ghost does not haunt QCD perturbation theory [32] is totally dependent upon the negative dissipation in the color conductivity. The resulting asymptotic freedom QCD vacuum instability might be equally embarrassing as the Landau ghost. But perhaps a QCD amplifier state is not unphysical.

Physical negative dissipation can occur when matter is pumped up by an external energy source. The resulting amplifier excited state can then return the energy when the matter decays back into the true ground state. In laboratory scattering experiments with large incident energies, an initial collision can excite the quark gluon constituents [33, 34] as well as pump up a small space-time piece of the vacuum. Within that small space-time collision region the QCD perturbation theory may have some validity while the vacuum is pumped into an excited state. However, the outgoing 
fragments of a collision must then contain energy in part due to the decay of the asymptotic free vacuum back to the true vacuum. The QCD view of strong interactions might then be flawed only to the extent that one has no clear picture of the true vacuum present at times well after the out going fragments (hadrons) enter into laboratory detectors.

[1] K. Wilson, Phys. Rev. D10, 2445 (1974).

[2] V. Gribov, "Orsay Lectures on Confinement", hep-ph/9905285 (1999).

[3] Y.N. Srivastava and A. Widom, Phys. Rev. D63, 077502 (2001).

[4] Y. Srivastava, S. Pacetti, G. Pancheri and A. Widom, in Proc. SLAC " $e^{+} e^{-}$Physics at intermediate Energies Workshop", Stanford, California, 30 April - 2 May 2001, ed. D. Bettoni, econf C0101430 T19 (2001), hep-ph/0106005

[5] A. Grau, S. Pacetti, G. Pancheri and Y. N. Srivastava, Nucl. Phys. B (Proc. Suppl.) 126, 84 (2004).

[6] D.V. Shirkov and I.L. Solovtsov, Phys. Rev. Lett. 79, 1209 (1997).

[7] A.V. Nesterenko, Mod. Phys. Lett. A15, 2401 (2000).

[8] V.B. Berestetskii, E.M. Lifshitz and L.P. Pitaevskii, "Quantum Electrodynamics", Ch.11, Pergamon Press, Oxford (1982).

[9] H.D. Politzer, Phys. Rev. Lett. 30, 1346 (1973).

[10] D. Gross and F. Wilczek, Phys. Rev. Lett. 30, 1343 (1973).

[11] F. Wilczek, Nature 397, 303 (1999).

[12] O. Panella, Y. N. Srivastava and A Widom, International Journal of Modern Physics A21, 3279-3288, (2006).

[13] N.F. Ramsey, Phys. Rev. 103, 20 (1956).

[14] M.J. Klein, Phys. Rev. 104, 589 (1956).

[15] A.L. Schalow and C.H. Townes, Phys. Rev. 112, 1940 (1958).

[16] J. Weber, Rev. Mod. Phys. 31, 681 (1959).

[17] T.H. Maiman, Nature 187, 493 (1960).

[18] R.J. Cohen, Rep. Prog. Phys. 52, 881 (1989)

[19] J.Herman and H. J. Habing, Phys. Rep. 124, 255 (1985).

[20] M. Eliztur, Rev. Mod. Phys. 54, 1225 (1982).

[21] H. Weaver, D.R. Williams H.N. Dieter and T.W. Lum, Nature 208, 29 (1965).

[22] G. K. Savvidy, Phys. Lett. B 71 (1977) 133.

[23] S. G. Matinyan and G. K. Savvidy, Nucl. Phys. B 134 (1978) 539.

[24] I. A. Batalin, S. G. Matinyan and G. K. Savvidy, Sov. J. Nucl. Phys. 26 (1977) 214 [Yad. Fiz. 26 (1977) 407].

[25] V. Branchina, P. Castorina, M. Consoli and D. Zappala, Phys. Lett. B 274 (1992) 404. 
[26] L.D. Landau and I.Ya. Pomeranchuk, Doklady AN SSSR 102, 489 (1955).

[27] S. Sivasubramanian, Y.N. Srivastava and A. Widom, Mod. Phys. Lett B16, 1201 (2002).

[28] K. Rajagopal and F. Wilczek, "The Condensed Matter Physics of QCD", Ch. 35 in "At the Frontier of Particle Physics / Handbook of QCD", M. Shifman Editor, World Scientific, Singapore (2001).

[29] N.N. Bogoliubov, A.A. Logunov and D.V. Shirkov, Sov. Phys. JETP 10, 574 (1959).

[30] V. Parihar, A. Widom and Y.N. Srivastava, Phys. Rev. C73, 017901 (2006).

[31] R.P. Feynman, Science 183, 601 (1974).

[32] T.D. Lee, "Particle Physics and Introduction to FieldTheory", Ch.17-19, Harwood Academic Publishers, London (1981).

[33] R.P. Feynman, Phys. Rev. Lett. 33, 1415 (1969).

[34] J. D. Bjorken and E. A. Paschos, Phys. Rev. 185, 1975 (1969). 